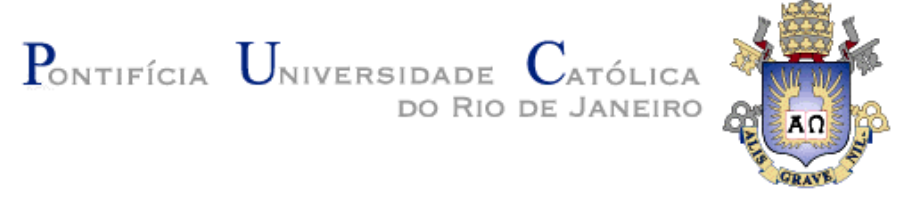

Katia Maria Carlos Rocha

\title{
Três Ensaios sobre a Metodologia de Apreçamento de Ativos utilizando Opções Reais
}

Tese de Doutorado

Tese apresentada como requisito parcial para obtenção do título de Doutor pelo Programa de Pós-Graduação em Engenharia Industrial da PUC-Rio.

Orientador: José Paulo Teixeira 


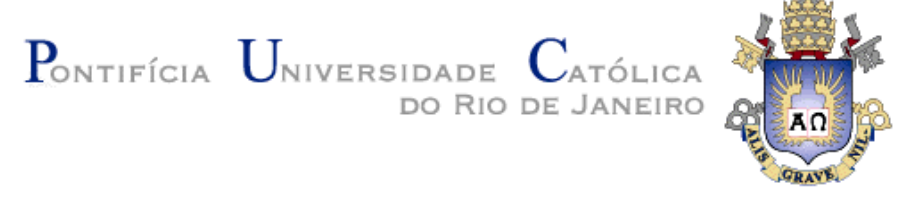

Katia Maria Carlos Rocha

\title{
Três Ensaios sobre a Metodologia de Apreçamento de Ativos utilizando Opções Reais
}

Tese apresentada como requisito parcial para obtenção do título de Doutor pelo Programa de Pós-Graduação em Engenharia Industrial da PUC-Rio. Aprovada pela Comissão Examinadora abaixo assinada.

\author{
José Paulo Teixeira \\ Orientador \\ Departamento de Engenharia Industrial - PUC-Rio \\ Luiz Eduardo Teixeira Brandão \\ IAG - PUC-Rio \\ Carlos Patrício Samanez \\ Departamento de Engenharia Industrial - PUC-Rio
}

Octávio Augusto Fontes Tourinho IPEA / BNDES / DAE UERJ

Antonio Carlos Figueiredo Pinto IAG - PUC-Rio

Andréa Maria Accioly Fonseca Minardi IBMEC - SP

Marco Antonio Oliveira FACC - UFRJ

José Eugênio Leal

Coordenador(a) Setorial do Centro Técnico Científico - PUC-Rio

Rio de Janeiro, 11 de dezembro de 2006 
Todos os direitos reservados. É proibida a reprodução total ou parcial do trabalho sem autorização da universidade, da autora e do orientador.

\section{Katia Maria Carlos Rocha}

Graduou-se em Engenheira Elétrica pela PUC-Rio em 1993 e desde 1997, trabalha como pesquisadora no Instituto de Pesquisa Econômica Aplicada (IPEA), fundação pública vinculada ao Ministério de Planejamento, Orçamento e Gestão. Seu trabalho consiste no desenvolvendo de estudos, relatórios e modelos econômicos para suporte de políticas públicas nas áreas de Risco País e Mercados Emergentes, Revisão Tarifária (Setores Distribuição e Telecomunicação), Regulação Econômica, Viabilidade Econômica da Geração, Apreçamento de Ativos (concessões florestais e de blocos exploratórios), Exploração e Produção de Petróleo, Otimização de Carteiras de Ativos Reais em E\&P e gás, Análise de Risco de Crédito.

Ficha Catalográfica

Rocha, Katia Maria Carlos

Três ensaios sobre a metodologia de apreçamento de ativos utilizando opções reais / Katia Maria Carlos Rocha ; orientador: José Paulo Teixeira. - 2006.

$109 \mathrm{f.} ; 30 \mathrm{~cm}$

Tese (Doutorado em Engenharia Industrial)-Pontifícia Universidade Católica do Rio de Janeiro, Rio de Janeiro, 2006.

Inclui bibliografia

1. Engenharia industrial - Teses. 2. Opções reais. 3. Telefonia fixa brasileira. 4. Tarifas de interconexão. 5. Incorporação imobiliária. 6. Gerenciamento de risco. 7. Mercados emergentes. 8. Spreads soberanos. 9. Probabilidade de default. I. Teixeira, José Paulo. II. Pontifícia Universidade Católica do Rio de Janeiro. Departamento de Engenharia Industrial. III. Título. 


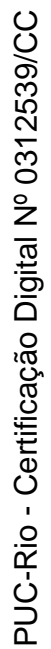

Aos meus pais Vilma Carlos Rocha e José Geraldo Rocha com muito amor 


\section{Agradecimentos}

Ao meu orientador José Paulo Teixeira pelo constante incentivo, orientação, sabedoria e carinho antes durante e após essa dissertação.

Aos professores Tara Keshar Nanda Baidya, Carlos Patrício Samanez, Paulo Henrique Soto Costa pelos formidáveis anos de convívio, parceria e debates.

Ao professor Nélio Domingues Pizzolato, que desde a graduação depositou confiança na oportunidade de integrar o quadro complementar do Departamento de Engenharia Industrial desde 2002.

À Isabel, Celi, Fernanda, Claudia, Gilvan e Eduardo pela paciência, ânimo e constante doação nesse excelente convívio de anos.

À PUC pelo auxílio através da bolsa integral.

Ao IPEA pelo tempo concedido aos estudos 


\section{Resumo}

Rocha, Katia Maria Carlos; Teixeira, José Paulo. Três Ensaios sobre a Metodologia de Apreçamento de Ativos utilizando Opções Reais. Rio de Janeiro, 2006. 109p. Tese de Doutorado - Departamento de Engenharia Industrial, Pontifícia Universidade Católica do Rio de Janeiro.

A dissertação apresenta três ensaios econômicos onde a abordagem de opções reais faz-se mister seja na definição de políticas regulatórias, estratégias de investimentos ou apreçamento de risco soberano. O primeiro ensaio, toma como premissa a nova regulação orientada a custos da interconexão de redes de telecomunicações e propõe ajustes no cálculo da remuneração de capital da telefonia fixa local. O modelo proposto estabelece o mark-up sobre o custo médio ponderado do capital (WACC) a ser aplicado nos novos contratos de concessão, levando-se em conta a opção de acesso disponibilizada pela operadora de STFC aos entrantes. O ensaio inova ao incorporar ao modelo de opções o impacto de mudanças de paradigmas tecnológicos que ocasionam saltos negativos na demanda da concessionária. Os resultados apontam para robustez do mark-up em relação a alterações nos parâmetros básicos do modelo (tráfego fixo-fixo, fixo-móvel e choques negativos de demanda), e apontam para um mark-up inferior a $1 \%$. O segundo ensaio analisa estratégias de investimentos em incorporação imobiliária, setor que envolve baixa liquidez, lento payback, e apresenta diversas incertezas econômicas relacionadas à demanda de mercado, preço por metro quadrado e custo do terreno. O ensaio analisa estratégias de lançamentos simultâneos e seqüenciais de empreendimentos imobiliários; o primeiro envolvendo um menor custo de construção, associado, porém, a uma maior incerteza nos resultados. O lançamento seqüencial apresenta características semelhantes a opções reais por embutir uma série de oportunidades quanto à aquisição de informações, adiamento e abandono do projeto. Apresenta-se o estudo de caso de uma incorporação na cidade do Rio de Janeiro, identificando-se a estratégia ótima bem como o preço máximo a ser pago pelo terreno. O lançamento seqüencial agrega valor adicional de $10 \%$ ao projeto além de diminuir a exposição ao risco do incorporador em mais de metade se comparado à metodologia tradicional de fluxo de caixa descontado. Finalmente, o terceiro ensaio recai sobre risco soberano e propõe um modelo estrutural a partir da teoria de opções e ativos contingentes para analisar a estrutura a termo de quatro países emergentes (Brasil, México, Rússia e Turquia) que representaram, em média, $54 \%$ do índice EMBIG do JPMorgan no período de 2000-2005. A taxa de câmbio real, modelada 
como um processo de difusão simples, é considerada como indicativa de default. $\mathrm{O}$ modelo calibrado indica que no período, o mercado sistematicamente sub-apreçou os títulos do Brasil em 100 pontos base na média, enquanto para México, Rússia e Turquia apreçou corretamente os spreads soberanos. O ensaio fornece ainda a probabilidade implícita de default do emissor, variável fundamental para o apreçamento dos derivativos de crédito, mercado que cresceu vertiginosamente após a crise da Ásia e Rússia, passando de US\$ 180 bilhões de dólares em 1996 para um valor esperado de US\$20 trilhões ao final de 2006. Este mercado é reconhecido como responsável por conter os efeitos contágios e manter a estabilidade no mercado financeiro em crises recentes como a da WorldCom, Parmalat, Enron entre outros.

\section{Palavras - chave}

Opções Reais; Telefonia Fixa Brasileira; Tarifas de Interconexão; Incorporação Imobiliária; Gerenciamento de Risco; Mercados Emergentes; Spreads Soberanos; Probabilidade de Default. 


\section{Abstract}

Rocha, Katia Maria Carlos, Teixeira, José Paulo. Three Essays on Asset Pricing applying Real Options Methodology. Rio de Janeiro, 2006. 109p. Ph.D. Thesis. Industrial Engineering Department. Pontifical Catholic University of Rio de Janeiro.

The dissertation presents three economic essays examining situations where the real options approach can be useful in the definition of regulatory policies, investment strategies and pricing of sovereign risk. The first essay considers the new regulation oriented to interconnection costs of telecommunications networks and proposes adjustments in calculating the return on capital invested in local fixed telephone service. The proposed model establishes the mark-up on the weighted average cost of capital (WACC) to be applied to new concession contracts, taking into account the access option provided by the fixed operator to entrants. The essay innovates by incorporating to the options model the impact of changes in technological paradigms that cause the concessionaire's demand to fall. The results indicate the robustness of the mark-up in relation to alterations in the model's basic parameters (fixed-fixed and fixed-mobile traffic and negative demand shocks), and mark-up was estimated to be under $1 \%$. The second essay analyzes investment strategies in real estate development, a sector that involves low liquidity, slow payback and various economic uncertainties related to market demand, price per square meter and land cost. The essay analyzes strategies for simultaneous and sequential launch of real estate projects. The first involves lower construction cost, but comes associated with more uncertain results. Sequential launch presents characteristics similar to real options because it has a series of built-in opportunities regarding the acquisition of information and delay or abandonment of the project. We present a case study of a development in the city of Rio de Janeiro, identifying the optimal strategy and the maximum land cost. Sequential launch aggregates $10 \%$ extra value to the undertaking, besides reducing the developer's risk exposure by over half in comparison with the traditional discounted cash flow method. Finally, the third essay examines sovereign risk and proposes a model from the theory of options and contingent assets to analyze the term structure of four emerging countries (Brazil, Mexico, Russia and Turkey) that together represented on average 54\% of JPMorgan's EMBIG index in the 2000-2005 period. The real exchange rate, modeled as a simple diffusion process, is considered as indicative of default. The calibrated model indicates that in the period studied, the market systematically underpriced Brazilian bonds by an average of 100 basis points, while for Mexico, Russia and Turkey it fairly 
priced the sovereign debt. The essay also provides the implicit probability of the issuer's default, a fundamental variable for pricing credit derivatives, a market that has grown at a dizzying pace since the Asian and Russian crises, rising from US\$ 180 billion in 1996 to an expected value of US\$20 trillion at the end of 2006 . This market is recognized as being responsible for containing the contagious effects and maintaining the stability of the financial market in recent crises, such as the corporate meltdowns of WorldCom, Parmalat and Enron, among others.

\section{Keywords}

Real Options, Brazilian Telecommunications Sector, Access Pricing, Real Estate, Risk Management, Emerging Markets, Sovereign Spreads, Default Probability. 


\section{Sumário}

1 Introdução 14

2 Opções de Acesso e a Tarifa de Interconexão (TU-RL) 20

2.1 Introdução 21

2.2 Revisão da Literatura 22

2.3 Arcabouço Legal e Regulatório 24

2.4 Aspectos Tecnológicos do Setor 25

2.4.1 Crescimento do Tráfego de Dados 26

2.4.2 Evolução das Arquiteturas de Rede 26

2.4.3 Novos Serviços - Choques Tecnológicos 27

2.5 Opções Reais e o Mark-Up sobre o WACC 28

2.5.1 A Opção de Investimento 30

2.5 .2 Estimativas 33

2.6 Conclusão 41

2.7 Referências Bibliográficas $\quad 42$

2.8 Apêndice A: A Demanda pelo Serviço de Telefonia Fixa 47

2.9 Apêndice B: A Volatilidade dos Retornos do Projeto. 48

2.10 Apêndice C: Modelo Brasileiro de Interconexão. 49

3 Opções Seqüenciais e a Incorporação Imobiliária 59

$\begin{array}{lll}3.1 & \text { Introdução } & 60\end{array}$

3.2 O Setor de Incorporação Imobiliária 62

3.3 A Viabilidade Econômica da Incorporação e as Opções Reais 65

3.4 Estudo de Caso: Incorporação na Cidade do Rio de Janeiro 69

3.4.1 Resultados 72

$\begin{array}{lll}3.5 & \text { Conclusão } & 76\end{array}$

$\begin{array}{lll}3.6 & \text { Referências Bibliográficas } & 77\end{array}$

3.7 Apêndice A: Aproximação de Barone-Adesi \& Whaley 79

3.8 Apêndice B: Probabilidade de Exercício de uma Opção Americana 80

3.9 Apêndice C: Indicadores de Performance em Incorporação 81

4 Opções de Default e os Spreads Soberanos 83

4.1 Introdução 84 
$\begin{array}{lll}4.2 & \text { O Modelo } & 87\end{array}$

4.3 Dados 88

$\begin{array}{lll}4.4 & \text { Resultados } & 91\end{array}$

4.5 Conclusão 95

4.6 Referências Bibliográficas 96

4.7 Apêndice: Distribuição de Primeira Passagem de Tempo 98

5 Conclusão e Futuras Recomendações 99

6 Bibliografia 102

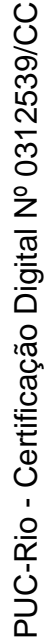




\section{Lista de figuras}

Figura 2.1 Distribuição de Probabilidade: Pulsos Faturados e VC1 por TMS 37

Figura 2.2 - Valor da Opção e o Exercício Ótimo 38

Figura 2.3 - Custo de Capital x Volatilidade (Choque Forte) 40

Figura 2.4 - Custo de Capital x Magnitude dos Choques (1choque/5 anos) 40

Figura A1 - Pulsos por Terminal Médio em Serviço $\quad 47$

Figura B1 - Distribuição dos Retornos do Projeto 48

Figura C1 - Valor pago e recebido na interconexão 56

Figura 3.1 - Processo de Decisão Estático. $\quad 65$

Figura 3.2 - Processo de Decisão Seqüencial. $\quad 66$

$\begin{array}{ll}\text { Figura } 3.3 \text { - Velocidade de Vendas (\%). } & 67\end{array}$

Figura 3.4 - Unidades Disponíveis e Escrituradas 2004.

Figura 3.5 - Financiamento Bancário e Lançamentos Imobiliários $2004 \quad 70$

Figura 3.6 - Preços de Vendas em mil Reais. $\quad 71$

Figura 3.7 - Lançamento Simultâneo vs. Seqüencial (R\$ Milhões). 72

Figura 3.8 - Prêmio da Opção - Segundo Lançamento (R\$ Milhões). 73

Figura 3.9 - Estratégia de Investimento Ótimo - Segundo Lançamento. 74

Figura 3.10. Distribuição de $\mathrm{VPL}_{1}$ - Lançamento Simultâneo. 75

Figura 3.11. Distribuição de $\mathrm{VPL}_{2}$ - Lançamento Seqüencial. 75

Figura 4.1 - Performance do EMBIG 84

Figura 4.2 - Spreads Soberanos $\quad 89$

Figura 4.3 - Composição do EMBIG \% 89

Figura 4.4 - Taxas de Câmbio Real $\quad 90$

Figura 4.5 - Volatilidade Anual $\quad 90$

Figura 4.6 - Spreads Previstos x Observados $\quad 91$

Figura 4.7 - Erro Médio Quadrático do Modelo 92

Figura 4.8 - Probabilidade Implícita de Default $\quad 94$ 


\section{Lista de tabelas}

Tabela 1-1 Opção Real vs. Financeira 18

Tabela 2-1 Projeção do Fluxo de Caixa 35

$\begin{array}{ll}\text { Tabela 2-2 Custo de Capital Corrigido } & 39\end{array}$

Tabela 3-1 Composição dos Empréstimos Imobiliários 2004

Tabela 3-2 Financiamento Imobiliário (\% PIB) 62

Tabela 3-3 Retornos Anuais - Estimação $\quad 72$

Tabela C1 Indicadores de Performance $\quad 81$

Tabela 4-1 Erro Médio Quadrático (\%) 93

Tabela 4-2 Spreads Sobre-Estimados $\quad 94$

Tabela 4-3 Probabilidade Cumulativa de Deafult 95 